\title{
Two Triterpenoid Saponins from Neonauclea sessilifolia
}

\author{
Atsuko Itoh, ${ }^{a}$ Takao TAnahashi, ${ }^{*, a}$ Naotaka NAgakura, ${ }^{a}$ and Toyoyuki Nishi ${ }^{b}$ \\ ${ }^{a}$ Kobe Pharmaceutical University; Higashinada-ku, Kobe 658-8558, Japan: and ${ }^{b}$ The Nippon Shinyaku Institute for \\ Botanical Research; Yamashina-ku, Kyoto 607-8182, Japan. Received July 3, 2003; accepted August 8, 2003
}

\begin{abstract}
From the dried roots of Neonauclea sessilifolia (Rubiaceae), two new triterpenoid saponins, 3-O- $\beta$-D-

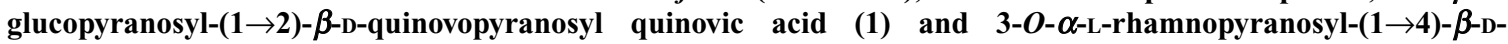
quinovopyranosyl pyrocincholic acid $28-O$ - $\beta$-D-glucopyranosyl-(1 $\rightarrow 6)$ - $\beta$-D-glucopyranosyl ester (2), were isolated, together with five known saponins. The structures of the new saponins were determined by spectroscopic and chemical means.
\end{abstract}

Key words Neonauclea sessilifolia; Rubiaceae; roots; structure elucidation; triterpenoid saponin

In the course of our phytochemical studies on glycosidic constituents of the plants belonging to Rubiaceae, we recently examined the roots of Neonauclea sessilifolia (Roxв.) MERR. and isolated several unique chromone-secoiridoid glycosides and indole alkaloid glycosides. ${ }^{1)}$ Further investigation of this plant material led us to isolate two new triterpenoid saponins, $\mathbf{1}$ and $\mathbf{2}$, along with five known triterpenoid saponins, 3- $O-\beta$-D-glucopyranosyl quinovic acid, ${ }^{2)} 3-O-\beta$-Dquinovopyranosyl quinovic acid (3), ${ }^{3)} 3-O-\beta$-D-glucopyranosyl-( $(1 \rightarrow 4)-\alpha$-L-rhamnopyranosyl quinovic acid ${ }^{4)} 3-O-\alpha$-Lrhamnopyranosyl-( $1 \rightarrow 2)-\beta$-D-glucopyranosyl-( $(1 \rightarrow 3)-\beta$-Dglucuronopyranosyl oleanolic acid $28-O$ - $\beta$-D-glucopyranosyl ester, ${ }^{5)}$ and 3- $O-\alpha$-L-rhamnopyranosyl-( $\left.1 \rightarrow 2\right)-\beta$-D-glucopyranosyl-( $1 \rightarrow 3)-6-O$-methyl- $\beta$-D-glucuronopyranosyl oleanolic acid 28-O- $\beta$-D-glucopyranosyl ester. ${ }^{5}$

Saponin 1 was isolated as a colorless crystalline solid, $\mathrm{mp}$ $220-222^{\circ} \mathrm{C}$. Its high resolution secondary ion mass spectrum (HR-SI-MS) showed a pseudomolecular ion at $\mathrm{m} / \mathrm{z}$ 793.4386, indicating a molecular formula $\mathrm{C}_{42} \mathrm{H}_{66} \mathrm{O}_{14}$. Acid hydrolysis of 1 afforded D-glucose and D-quinovose which were identified by GLC analysis of their thiazolizine derivatives. ${ }^{6}$ The ${ }^{1} \mathrm{H}-\mathrm{NMR}$ spectral features of $\mathbf{1}$ showed analogy to those of 3-O- $\beta$-D-quinovopyranosyl quinovic acid (3), a major saponin of this plant, except that $\mathbf{1}$ showed the signals due to an additional glucose moiety. Two anomeric proton signals at $\delta 4.39(\mathrm{~d}, J=7.5 \mathrm{~Hz})$ and $4.66(\mathrm{~d}, J=7.5 \mathrm{~Hz})$ indicated $\beta$-linkage of quinovopyranose and glucopyranose units in $\mathbf{1}$. The ${ }^{13} \mathrm{C}$-NMR spectrum of $\mathbf{1}$ showed, besides 12 signals due to two hexose moieties, 30 carbon signals which were observed in the nearly identical frequencies of those of 3-O$\beta$-D-quinovopyranosyl quinovic acid (3), indicating a quinovic acid as an aglycone unit and the glycosidation at $\mathrm{C}$ 3. ${ }^{1} \mathrm{H}$-Detected heteronuclear multiple-bond connectivity (HMBC) correlations between $\mathrm{H}-1^{\prime}$ of quinovose and $\mathrm{C}-3$ ( $\delta$ 91.4), and between $\mathrm{H}-1^{\prime \prime}$ of glucose and $\mathrm{C}-2^{\prime}$ of quinovose $(\delta$ $81.3)$ showed the attachment of 2- $O-\beta$-D-glucopyranosyl- $\beta$ D-quinovopyranose to $\mathrm{C}-3$ of quinovic acid. The linkage of a terminal glucose to C-2' of quinovosyl moiety was supported by a comparative study of the ${ }^{13} \mathrm{C}-\mathrm{NMR}$ spectra of $\mathbf{1}$ and $\mathbf{3}$ which showed the downfield shift of $\mathrm{C}-2^{\prime}$ of quinovose moiety $(+5.4 \mathrm{ppm})$ and the upfield shift of $\mathrm{C}-1^{\prime}$ and $\mathrm{C}-2^{\prime}(-1.3$ and $-0.3 \mathrm{ppm}$, respectively). Consequently, glycoside 1 was deduced to be 3-O- $\beta$-D-glucopyranosyl-( $1 \rightarrow 2)$ - $\beta$-D-quinovopyranosyl quinovic acid.

Saponin 2 was obtained as a white powder, $\mathrm{C}_{53} \mathrm{H}_{86} \mathrm{O}_{21}$, $[\alpha]_{\mathrm{D}}-43^{\circ}$. Acid hydrolysis of 2 gave D-glucose, D- quinovose, and L-rhamnose. Its ${ }^{1} \mathrm{H}$ - and ${ }^{13} \mathrm{C}$-NMR spectra revealed the signals due to two $\beta$-glucopyranose, a $\beta$ quinovopyranose and an $\alpha$-rhamnopyranose unit. Furthermore, its ${ }^{13} \mathrm{C}$-NMR showed 29 carbon signals as an aglycone moiety involving six tertiary methyl groups, a carbonyl group and two quaternary olefinic carbon signals. The NMR spectral features were closely similar to those of 3-O- $\beta$-Dquinovopyranosyl pyrocincholic acid $28-O-\beta$-D-glucopyranosyl-( $(1 \rightarrow 6)-\beta$-D-glucopyranosyl ester $\left.(4),{ }^{7}\right)$ except for the signals arising from an additional $\alpha$-rhamnose moiety. The downfield shift of C-4' and upfield shift of C-3' and C-5' of 2 , relative to 4 , were ascribed to the glycosidation of $4^{\prime}$-hydroxy group in the quinovose moiety. The attachment of 4-O$\alpha$-L-rhamnopyranosyl- $\beta$-D-quinovopyranose unit to C-3 of pyrocincholic acid and the ester linkage of the carboxyl group at $\mathrm{C}-28$ of pyrocincholic acid with $6-O-\beta$-D-glucopyranosyl- $\beta$-D-glucopyranose unit were further confirmed by detailed two dimensional (2D)-NMR experiments, which showed HMBC interactions between $\mathrm{H}-3$ and $\mathrm{C}-1^{\prime}$ of quinovose, between $\mathrm{H}-1^{\prime}$ of quinovose and $\mathrm{C}-3$, between $\mathrm{H}-$ $4^{\prime}$ of quinovose and $\mathrm{C}-1^{\prime \prime}$ of rhamnose, between $\mathrm{H}-1^{\prime \prime}$ of rhamnose and C-4' of quinovose, between $\mathrm{H}-1^{\prime \prime \prime}$ of inner glucose and C-28, between $\mathrm{H}-6^{\prime \prime \prime}$ of inner glucose and C-1"'"' of terminal glucose, and between $\mathrm{H}-1^{\prime \prime \prime \prime}$ of terminal glucose and C-6"' of inner glucose. Accordingly, glycoside 2 was assigned to $3-O-\alpha$-L-rhamnopyranosyl-( $1 \rightarrow 4)-\beta$-D-quinovopyranosyl pyrocincholic acid $28-O-\beta$-D-glucopyranosyl-( $1 \rightarrow 6)$ $\beta$-D-glucopyranosyl ester.

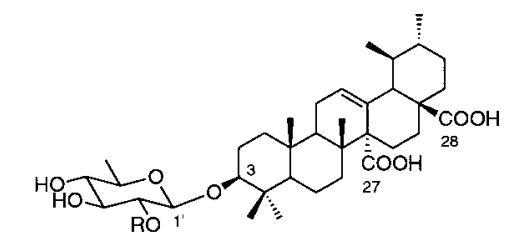

1: $\mathrm{R}=\beta$-D-Glc

3: $R=H$

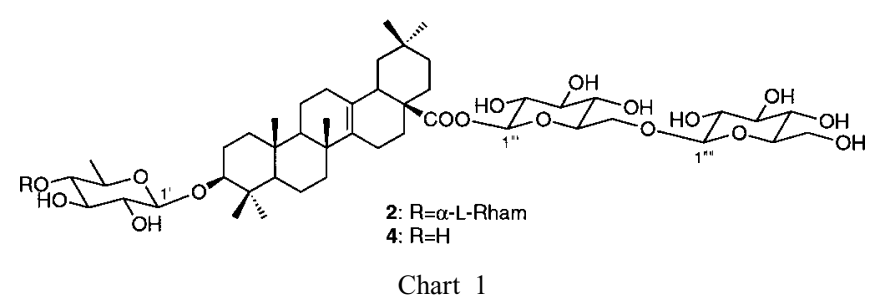

* To whom correspondence should be addressed. e-mail: tanahash@kobepharma-u.ac.jp 
Table 1. ${ }^{13} \mathrm{C}-\mathrm{NMR}$ Spectral Data of $\mathbf{1}-\mathbf{4}$

\begin{tabular}{|c|c|c|c|c|c|c|c|c|c|c|c|}
\hline $\mathrm{C}$ & $\mathbf{1}^{a)}$ & $2^{a)}$ & $2^{b)}$ & $3^{c)}$ & $4^{d)}$ & $\mathrm{C}$ & $\mathbf{1}^{a)}$ & $2^{a)}$ & $2^{b)}$ & $3^{c)}$ & $4^{d)}$ \\
\hline 1 & 39.9 & 39.5 & 38.4 & 39.9 & 38.5 & & 3-O-Qui & 3-O-Qui & 3-O-Qui & 3-O-Qui & 3-O-Qui \\
\hline 2 & 27.2 & 27.2 & 26.7 & 27.1 & 26.9 & $1^{\prime}$ & 105.2 & 106.4 & 106.4 & 106.5 & 106.8 \\
\hline 3 & 91.4 & 90.8 & 89.1 & 90.7 & 89.0 & $2^{\prime}$ & 81.3 & 76.0 & 76.0 & 75.9 & 76.0 \\
\hline 4 & 40.4 & 40.3 & 39.6 & 40.1 & 39.6 & $3^{\prime}$ & 78.2 & 76.7 & 76.5 & 77.9 & 78.0 \\
\hline 5 & 56.9 & 57.1 & 55.7 & 56.9 & 55.8 & $4^{\prime}$ & 76.9 & 85.8 & 84.9 & 77.0 & 77.0 \\
\hline 6 & 19.3 & 18.9 & 18.7 & 19.3 & 18.8 & $5^{\prime}$ & 72.9 & 71.9 & 71.4 & 73.0 & 72.8 \\
\hline 7 & 38.0 & 40.7 & 39.7 & 38.0 & 39.7 & $6^{\prime}$ & 18.2 & 18.5 & 18.6 & 18.2 & 18.9 \\
\hline 8 & 40.7 & 38.2 & 38.0 & 40.7 & 38.1 & & $2^{\prime}-O$-Glc & $4^{\prime}-O-\mathrm{Rha}$ & $4^{\prime}-O-\mathrm{Rha}$ & & \\
\hline 9 & 48.0 & 57.8 & 56.5 & 48.0 & 56.5 & $1^{\prime \prime}$ & 104.5 & 103.2 & 103.0 & & \\
\hline 10 & 37.8 & 38.9 & 37.2 & 37.8 & 37.2 & $2^{\prime \prime}$ & 76.3 & 72.4 & 72.5 & & \\
\hline 11 & 23.9 & 19.5 & 18.1 & 23.8 & 18.1 & $3^{\prime \prime}$ & 77.9 & 72.2 & 72.7 & & \\
\hline 12 & 130.4 & 32.1 & 32.0 & 130.4 & 32.1 & $4^{\prime \prime}$ & 71.9 & 73.7 & 73.9 & & \\
\hline 13 & 133.9 & 131.2 & 130.3 & 133.9 & 130.3 & $5^{\prime \prime}$ & 78.4 & 70.7 & 70.5 & & \\
\hline 14 & 57.3 & 137.9 & 136.9 & 57.3 & 137.0 & $6^{\prime \prime}$ & 63.1 & 17.8 & 18.5 & & \\
\hline 15 & 26.5 & 21.6 & 21.0 & 26.5 & 21.0 & & & $28-O-G l c$ & $28-O-G l c$ & & $28-O-G l c$ \\
\hline 16 & 25.7 & 24.4 & 24.1 & 25.7 & 24.8 & $1^{\prime \prime \prime}$ & & 95.7 & 95.7 & & 95.7 \\
\hline 17 & * & 46.6 & 45.7 & * & 45.7 & $2^{\prime \prime \prime}$ & & 74.0 & 74.0 & & 75.2 \\
\hline 18 & 55.5 & 40.2 & 39.5 & 55.5 & 39.5 & $3^{\prime \prime \prime}$ & & 78.2 & $78.8^{e)}$ & & 78.4 \\
\hline 19 & 40.4 & 42.4 & 41.5 & 40.4 & 41.5 & $4^{\prime \prime \prime}$ & & 71.0 & 71.0 & & 71.6 \\
\hline 20 & 38.3 & 31.4 & 30.6 & 38.3 & 30.9 & $5^{\prime \prime \prime}$ & & 77.8 & 78.0 & & 78.4 \\
\hline 21 & 31.2 & 35.1 & 34.3 & 31.2 & 34.4 & $6^{\prime \prime \prime}$ & & 69.6 & 69.5 & & 69.5 \\
\hline 22 & 37.6 & 32.8 & 31.2 & 37.6 & 32.1 & & & $6^{\prime \prime \prime}-O-G l c$ & $6^{\prime \prime \prime}-O-G l c$ & & $6^{\prime \prime \prime}-O-\mathrm{Glc}$ \\
\hline 23 & 28.4 & 28.4 & 28.1 & 28.5 & 28.2 & $1^{\prime \prime \prime \prime}$ & & 104.7 & 105.3 & & 105.3 \\
\hline 24 & 16.8 & 16.7 & 16.7 & 17.0 & 16.7 & $2^{\prime \prime \prime \prime}$ & & 75.2 & 75.2 & & 74.1 \\
\hline 25 & 17.0 & 17.2 & 16.7 & 16.9 & 16.7 & $3^{\prime \prime \prime \prime}$ & & 78.0 & $78.4^{e)}$ & & 78.8 \\
\hline 26 & 18.2 & 21.2 & 20.9 & 18.2 & 20.9 & $4^{\prime \prime \prime \prime}$ & & 71.6 & 71.6 & & 71.0 \\
\hline 27 & 179.0 & - & - & 179.0 & - & $5^{\prime \prime \prime \prime}$ & & 78.0 & $78.5^{e)}$ & & 78.5 \\
\hline 28 & 181.6 & 178.2 & 176.7 & 181.6 & 176.8 & $6^{\prime \prime \prime \prime}$ & & 62.8 & 62.7 & & 62.7 \\
\hline 29 & 19.1 & 33.0 & 32.3 & 19.1 & 32.4 & & & & & & \\
\hline 30 & 21.5 & 25.2 & 25.0 & 21.5 & 25.0 & & & & & & \\
\hline
\end{tabular}

a) Measured in $\mathrm{CD}_{3} \mathrm{OD}$ at $125 \mathrm{MHz}$. b) Measured in pyridine- $d_{5}$ at $125 \mathrm{MHz}$. c) Measured in $\mathrm{CD}_{3} \mathrm{OD}$ at $75 \mathrm{MHz}$. d) Measured in pyridine- $d_{5}$ at $75 \mathrm{MHz}$. Data taken from ref. 7. e) Values are interchangeable. * Overlapped with solvent signal.

While triterpenoid saponins have so far been isolated from the plant species of the genera Nauclea and Adina (Rubiaceae), the present work gave the first example of isolation of triterpenoid saponins $\mathbf{1}$ and $\mathbf{2}$ from the Neonauclea species of the same family.

\section{Experimental}

IR spectra were recorded on a Shimadzu FTIR-8200 spectrophotometer Optical rotations were measured on a Jasco DIP-370 digital polarimeter. ${ }^{1} \mathrm{H}$ $(500 \mathrm{MHz})$ and ${ }^{13} \mathrm{C}-(125 \mathrm{MHz}) \mathrm{NMR}$ spectra were recorded on a Varian VXR-500 spectrometer with TMS as an internal standard. MS and HR-MS were obtained with a Hitachi M-4100 mass spectrometer. Glycerol was used for SI-MS and HR-SI-MS as the matrix. Medium pressure liquid chromatography (MPLC) was carried out with Wakogel 40C18. TLC was performed on precoated Kieselgel $60 \mathrm{~F}_{254}$ plates (Merck). HPLC was performed using a Waters system (600E System Controller, 486 Tunable Absorbance Detector). GLC was carried out on a Shimadzu GC-18A equipped with FID.

Isolation of Saponins The roots of Neonauclea sessilifolia were collected at Surat Thani, Thailand. A voucher specimen (NST-592) is deposited in the laboratory of Nippon Shinyaku Institute for Botanical Research. Dried roots $(1.58 \mathrm{~kg})$ of $N$. sessilifolia were extracted with hot $\mathrm{MeOH}$ and the extract was fractionated as previously reported in ref. 1. Fraction 9 in ref. 1 was purified by a combination of $\mathrm{SiO}_{2} \mathrm{CC}$ with $\mathrm{MeOH}-\mathrm{CHCl}_{3}(7: 93-$ $2: 3$ ), prep. HPLC ( $\mu$ Bondasphere $5 \mu \mathrm{C} 18-100 \AA, \mathrm{MeOH}-\mathrm{H}_{2} \mathrm{O}, 3: 1,4: 1$, $3: 2)$ and prep. TLC $\left(\mathrm{CHCl}_{3}-\mathrm{MeOH}, 4: 1,7: 3\right.$; AcOEt- $\mathrm{C}_{6} \mathrm{H}_{6}-\mathrm{EtOH}$, $4: 1: 2)$ to afford 3 - $O-\beta$-D-glucopyranosyl quinovic acid $(13.5 \mathrm{mg}), 3$ $(94.4 \mathrm{mg}), \quad 3-O-\beta$-D-glucopyranosyl-( $1 \rightarrow 4)-\alpha$-L-rhamnopyranosyl quinovic acid $(9.2 \mathrm{mg}), \mathbf{1}(19.6 \mathrm{mg}), 2$ ( $8.4 \mathrm{mg}), 3-O-\alpha$-L-rhamnopyranosyl-( $1 \rightarrow 2)-\beta$ D-glucopyranosyl-( $1 \rightarrow 3)$-6- $O$-methyl- $\beta$-D-glucuronopyranosyl oleanolic acid $28-O-\beta$-D-glucopyranosyl ester $(19.4 \mathrm{mg}$ ), and $3-O-\alpha$-L-rhamnopyranosyl-( $1 \rightarrow 2)$ - $\beta$-D-glucopyranosyl-( $1 \rightarrow 3$ )- $\beta$-D-glucuronopyranosyl oleanolic acid $28-O-\beta$-D-glucopyranosyl ester $(65.8 \mathrm{mg})$.

3- $O$ - $\beta$-D-Glucopyranosyl-( $(\rightarrow 2)-\beta$-D-quinovocyranosyl Quinovic Acid (1): Colorless crystalline solid. mp $220-222^{\circ} \mathrm{C}\left(\mathrm{H}_{2} \mathrm{O}\right)$. $[\alpha]_{\mathrm{D}}^{22}+43^{\circ}(c=0.6$,
MeOH). IR ( $\mathrm{KBr}) \mathrm{cm}^{-1}: 3426,2928,1697,1074 .{ }^{1} \mathrm{H}-\mathrm{NMR}\left(\mathrm{CD}_{3} \mathrm{OD}\right) \delta$ : $0.74(1 \mathrm{H}, \mathrm{brd}, J=11.5 \mathrm{~Hz}, \mathrm{H}-5), 0.84\left(3 \mathrm{H}, \mathrm{s}, \mathrm{H}_{3}-24\right), 0.89\left(3 \mathrm{H}, \mathrm{s}, \mathrm{H}_{3}-26\right)$, $0.90\left(3 \mathrm{H}, \mathrm{d}, J=5.0 \mathrm{~Hz}, \mathrm{H}_{3}-29\right), 0.92\left(3 \mathrm{H}, \mathrm{d}, J=5.0 \mathrm{~Hz}, \mathrm{H}_{3}-30\right), 0.97(3 \mathrm{H}, \mathrm{s}$, $\left.\mathrm{H}_{3}-25\right), 1.04\left(3 \mathrm{H}, \mathrm{s}, \mathrm{H}_{3}-23\right), 1.26\left(3 \mathrm{H}, \mathrm{d}, J=6.0 \mathrm{~Hz}, \mathrm{H}_{3}-6^{\prime}\right), 2.23(1 \mathrm{H}, \mathrm{dd}$, $J=11.0,5.0 \mathrm{~Hz}, \mathrm{H}-9), 2.25(1 \mathrm{H}, \mathrm{brd}, J=11.0 \mathrm{~Hz}, \mathrm{H}-18), 2.99(1 \mathrm{H}, \mathrm{t}$, $\left.J=9.0 \mathrm{~Hz}, \mathrm{H}-4^{\prime}\right), 3.12(1 \mathrm{H}, \mathrm{dd}, J=11.5,4.5 \mathrm{~Hz}, \mathrm{H}-3), 3.18(1 \mathrm{H}, \mathrm{t}, J=9.0 \mathrm{~Hz}$, $\left.\mathrm{H}-4^{\prime \prime}\right), 3.20\left(1 \mathrm{H}, \mathrm{dd}, J=9.0,7.5 \mathrm{~Hz}, \mathrm{H}-2^{\prime \prime}\right), 3.24(1 \mathrm{H}, \mathrm{ddd}, J=9.0,6.0,2.0 \mathrm{~Hz}$, H-5"), $3.29\left(1 \mathrm{H}, \mathrm{dq}, J=9.0,6.0 \mathrm{~Hz}, \mathrm{H}-5^{\prime}\right), 3.34\left(1 \mathrm{H}, \mathrm{t}, J=9.0 \mathrm{~Hz}, \mathrm{H}-3^{\prime \prime}\right)$, $3.49\left(1 \mathrm{H}, \mathrm{t}, J=9.0 \mathrm{~Hz}, \mathrm{H}-3^{\prime}\right), 3.56\left(1 \mathrm{H}, \mathrm{dd}, J=9.0,7.5 \mathrm{~Hz}, \mathrm{H}-2^{\prime}\right), 3.60(1 \mathrm{H}$, dd, $\left.J=11.5,6.0 \mathrm{~Hz}, \mathrm{H}-6^{\prime \prime}\right), 3.81\left(1 \mathrm{H}, \mathrm{dd}, J=11.5,2.0 \mathrm{~Hz}, \mathrm{H}-6^{\prime \prime}\right), 4.39(1 \mathrm{H}, \mathrm{d}$, $\left.J=7.5 \mathrm{~Hz}, \mathrm{H}-1^{\prime}\right), 4.66\left(1 \mathrm{H}, \mathrm{d}, J=7.5 \mathrm{~Hz}, \mathrm{H}-1^{\prime \prime}\right), 5.60(1 \mathrm{H}, \mathrm{dd}, J=5.0,2.0 \mathrm{~Hz}$, H-12). ${ }^{13} \mathrm{C}-\mathrm{NMR}$ : Table 1. Negative ion SI-MS $m / z$ : $793(\mathrm{M}-\mathrm{H})^{-}$, 587. HRSI-MS $m / z$ : 793.4386 (Calcd for $\mathrm{C}_{42} \mathrm{H}_{65} \mathrm{O}_{14}$ : 793.4377). $\mathrm{HMBC}$ : $\mathrm{H}-18$ to C28; $\mathrm{H}-1^{\prime}$ to $\mathrm{C}-3$; $\mathrm{H}-1^{\prime \prime}$ to $\mathrm{C}-2^{\prime}$.

$3-O-\alpha$-L-Rhamnopyranosyl-( $1 \rightarrow 4)-\beta$-D-quinovopyranosyl Pyrocincholic Acid $28-O-\beta$-D-Glucopyranosyl-( $1 \rightarrow 6)-\beta$-D-glucopyranosyl Ester (2): Amorphous powder. $[\alpha]_{\mathrm{D}}^{25}-43^{\circ}(c=0.5, \mathrm{MeOH})$. IR $(\mathrm{KBr}) \mathrm{cm}^{-1}: 3421$, 2941, 1734, 1636, 1065. ${ }^{1} \mathrm{H}-\mathrm{NMR}$ (pyridine- $\left.d_{5}\right) \delta: 0.78(1 \mathrm{H}, \quad$ brd, $J=12.0 \mathrm{~Hz}, \mathrm{H}-5), 0.81\left(3 \mathrm{H}, \mathrm{s}, \mathrm{H}_{3}-25\right), 0.90\left(3 \mathrm{H}, \mathrm{s}, \mathrm{H}_{3}-30\right), 0.90\left(3 \mathrm{H}, \mathrm{s}, \mathrm{H}_{3}-\right.$ 29), $0.95\left(3 \mathrm{H}, \mathrm{s}, \mathrm{H}_{3}-24\right), 1.04(1 \mathrm{H}, \mathrm{dd}, J=12.0,2.5 \mathrm{~Hz}, \mathrm{H}-9), 1.14(3 \mathrm{H}, \mathrm{s}$, $\left.\mathrm{H}_{3}-26\right), 1.21(1 \mathrm{H}$, br t, $J=13.0 \mathrm{~Hz}, \mathrm{H}-19), 1.31\left(3 \mathrm{H}, \mathrm{s}, \mathrm{H}_{3}-23\right), 1.41(3 \mathrm{H}, \mathrm{d}$, $\left.J=5.5 \mathrm{~Hz}, \mathrm{H}_{3}-6^{\prime}\right), 1.71\left(3 \mathrm{H}, \mathrm{d}, J=6.0 \mathrm{~Hz}, \mathrm{H}_{3}-6^{\prime \prime}\right), 1.89(1 \mathrm{H}$, br q, $J=12.0 \mathrm{~Hz}$, $\mathrm{H}-2), 2.77(1 \mathrm{H}, \mathrm{dd}, J=12.0,4.0 \mathrm{~Hz}, \mathrm{H}-18), 3.34(1 \mathrm{H}, \mathrm{dd}, J=12.0,4.0 \mathrm{~Hz}, \mathrm{H}-$ 3), $3.67\left(1 \mathrm{H}, \mathrm{dq}, J=9.0,5.5 \mathrm{~Hz}, \mathrm{H}-5^{\prime}\right), 3.70\left(1 \mathrm{H}, \mathrm{t}, J=9.0 \mathrm{~Hz}, \mathrm{H}-4^{\prime}\right), 3.90$ $\left(1 \mathrm{H}\right.$, ddd, $\left.J=9.0,5.0,2.0 \mathrm{~Hz}, \mathrm{H}-5^{\prime \prime \prime \prime}\right), 3.98\left(1 \mathrm{H}\right.$, br t $\left., J=8.0 \mathrm{~Hz}, \mathrm{H}-2^{\prime}\right), 4.02$ $\left(1 \mathrm{H}\right.$, br t $\left., J=8.5 \mathrm{~Hz}, \mathrm{H}-2^{\prime \prime \prime}\right), 4.08\left(1 \mathrm{H}\right.$, br t $\left., J=8.5 \mathrm{~Hz}, \mathrm{H}-3^{\prime}\right), 4.10$ (1H, ddd, $\left.J=9.5,4.5,2.0 \mathrm{~Hz}, \mathrm{H}-5^{\prime \prime \prime}\right), 4.14\left(1 \mathrm{H}\right.$, br t $\left., J=8.0 \mathrm{~Hz}, \mathrm{H}-2^{\prime \prime \prime}\right), 4.20(1 \mathrm{H}, \mathrm{brt}$, $\left.J=8.0 \mathrm{~Hz}, \mathrm{H}-3^{\prime \prime \prime \prime}\right), 4.22\left(1 \mathrm{H}\right.$, br t $\left., J=8.0 \mathrm{~Hz}, \mathrm{H}-3^{\prime \prime \prime}\right), 4.24(1 \mathrm{H}$, br t,$J=8.5 \mathrm{~Hz}$, H-4'"'), 4.35 ( $2 \mathrm{H}$, brt $\left., J=9.0 \mathrm{~Hz}, \mathrm{H}-4^{\prime \prime}, \mathrm{H}-4^{\prime \prime \prime}\right), 4.37$ (2H, m, H-6"', H-6"''"), $4.50\left(1 \mathrm{H}, \mathrm{dd}, J=11.5,2.0 \mathrm{~Hz}, \mathrm{H}-6^{\prime \prime \prime}\right), 4.54\left(1 \mathrm{H}, \mathrm{dd}, J=9.0,3.0 \mathrm{~Hz}, \mathrm{H}-3^{\prime \prime}\right)$, $4.64\left(1 \mathrm{H}\right.$, br s, H-2") $, 4.73\left(1 \mathrm{H}, \mathrm{dd}, J=11.0,2.0 \mathrm{~Hz}, \mathrm{H}-6^{\prime \prime \prime}\right), 4.78(1 \mathrm{H}, \mathrm{d}$, $\left.J=8.0 \mathrm{~Hz}, \mathrm{H}-1^{\prime}\right), 4.90\left(1 \mathrm{H}, \mathrm{dq}, J=9.5,6.0 \mathrm{~Hz}, \mathrm{H}-5^{\prime \prime}\right), 5.05(1 \mathrm{H}, \mathrm{d}, J=8.0 \mathrm{~Hz}$, $\left.\mathrm{H}-1^{\prime \prime \prime \prime}\right), 5.59\left(1 \mathrm{H}, \mathrm{d}, J=1.0 \mathrm{~Hz}, \mathrm{H}-1^{\prime \prime}\right), 6.27\left(1 \mathrm{H}, \mathrm{d}, J=8.0 \mathrm{~Hz}, \mathrm{H}-1^{\prime \prime \prime}\right) .{ }^{13} \mathrm{C}-$ NMR: Table 1. Negative ion SI-MS $m / z: 1057(\mathrm{M}-\mathrm{H})^{-}, 733$. HR-SI-MS $m / z$ : 1057.5571 (Calcd for $\mathrm{C}_{53} \mathrm{H}_{85} \mathrm{O}_{21}$ : 1057.5587). ROESY: H-3/H-1'; H- 
4'/H-1"; H-6"'/H-1"'". HMBC: H-18 to C-13, 14, 17, 19; H-16 to C-28; $\mathrm{H}_{3}-26$ to $\mathrm{C}-7,8,9,14$; $\mathrm{H}-3$ to $\mathrm{C}-1^{\prime} ; \mathrm{H}-1^{\prime}$ to $\mathrm{C}-3 ; \mathrm{H}-4^{\prime}$ to $\mathrm{C}-1^{\prime \prime}$; $\mathrm{H}-1^{\prime \prime}$ to $\mathrm{C}-4^{\prime} ; \mathrm{H}-1^{\prime \prime \prime}$ to $\mathrm{C}-28 ; \mathrm{H}_{2}-6^{\prime \prime \prime}$ to $\mathrm{C}-1^{\prime \prime \prime \prime} ; \mathrm{H}-1^{\prime \prime \prime \prime}$ to $\mathrm{C}-6^{\prime \prime \prime}$.

Acid Hydrolysis of Saponins 1 and 2 Each saponin (1 mg) was heated at $95{ }^{\circ} \mathrm{C}$ with dioxane $(0.5 \mathrm{ml})$ and $5 \% \mathrm{H}_{2} \mathrm{SO}_{4}(0.5 \mathrm{ml})$ for $1 \mathrm{~h}$. After neutralization with Amberlite IRA-400 ( $\mathrm{OH}^{-}$form), the reaction mixture was concentrated and the residue was passed through a Sep-Pak $\mathrm{C}_{18}$ cartridge with $\mathrm{H}_{2} \mathrm{O}$. The eluate was concentrated and the residue was treated with L-cysteine methyl ester hydrochloride $(1 \mathrm{mg})$ in pyridine $(0.125 \mathrm{ml})$ at $60{ }^{\circ} \mathrm{C}$ for $1 \mathrm{~h}$. The solution was then treated with $\mathrm{N}, \mathrm{O}$-bis(trimethylsilyl)trifluoroacetamide $(0.05 \mathrm{ml})$ at $60^{\circ} \mathrm{C}$ for $1 \mathrm{~h}$. The supernatant was applied to GLC; GLC conditions: column, Supelco $\mathrm{SPB}^{\mathrm{TM}}-1,30 \mathrm{~m} \times 0.25 \mathrm{~mm}$; column temperature, $230{ }^{\circ} \mathrm{C} ; \mathrm{N}_{2}$ flow rate, $0.8 \mathrm{ml} / \mathrm{min} ; t_{\mathrm{R}}$ of derivatives, D-glucose $13.1 \mathrm{~min}$, L-glucose $13.6 \mathrm{~min}$, D-quinovose $8.7 \mathrm{~min}$, L-rhamnose $9.0 \mathrm{~min}$. DGlucose and D-quinovose were detected from $\mathbf{1}$ and 2, and L-rhamnose was additionally detected from 2 .

Acknowledgements Our thanks go to Dr. M. Sugiura (Kobe Pharma- ceutical University) for NMR spectra and to Dr. K. Saiki (Kobe Pharmaceutical University) for MS measurements. This research was financially supported by the Kobe Pharmaceutical University Collaboration Fund.

\section{References}

1) Itoh A., Tanahashi T., Nagakura N., Nishi T., Phytochemistry, 62, 359-369 (2003).

2) Aquino R., De Simone F., Pizza C., Cerri R., De Mello J. F., Phytochemistry, 27, 2927-2930 (1988).

3) Ahmad V. U., Ghazala, Uddin S., Bano S., J. Nat. Prod., 53, 1193 1197 (1990).

4) Fang S.-Y., He Z.-S., Gao J.-H., Wang P., Phytochemistry, 39, 12411243 (1995).

5) Seshadri R., Rangaswami S., Indian J. Chem., 13, $447-452$ (1975).

6) Hara S., Okabe H., Mihashi K., Chem. Pharm. Bull., 34, 1843-1845 (1986).

7) Um B.-H., Weniger B., Lobstein A., Pouplin T., Polat M., Aragon R., Anton R., J. Nat. Prod., 64, 1588-1589 (2001). 